\section{Selective assembly of HIV-1 Vif-Cul5-ElonginB-ElonginC E3 ubiquitin ligase complex through a novel SOCS box and upstream cysteines}

Yunkai Yu, ${ }^{1,4}$ Zuoxiang Xiao, ${ }^{2,4}$ Elana S. Ehrlich, ${ }^{1}$ Xianghui $\mathrm{Yu}^{3}$ and Xiao-Fang $\mathrm{Yu}^{1,2,5}$

${ }^{1}$ Department of Molecular Microbiology and Immunology, Johns Hopkins University Bloomberg School of Public Health, Baltimore, Maryland 21205, USA; ${ }^{2}$ Zhejiang University, Zhejiang 310009, China; ${ }^{3}$ Jilin University, Jilin 130023, China

APOBEC3G, which induces hypermutations in newly synthesized viral DNA, is suppressed by HIV-1 Vif, acting through Cul5-ElonginB-ElonginC E3 ubiquitin ligase. We have now characterized a novel SOCS box in HIV-1 Vif that mediates its interaction with ElonginC. In this SOCS box, alanine replaces the consensus cysteine in the previously identified SOCS box. This new motif was necessary but insufficient for interaction with Cul5-ElonginB-ElonginC, as two highly conserved Cys residues outside the SOCS box were required to interact with Cul5 but not ElonginC. Therefore, selective assembly with Cul5 versus Cul2 E3 may require protein interfaces besides the SOCS-box-ElonginC interaction.

Received August 16, 2004; revised version accepted September 24, 2004.

APOBEC3G mediates antiviral activity by inducing hypermutations in newly synthesized viral minus-strand DNA (Sheehy et al. 2002; Harris et al. 2003; Lecossier et al. 2003; Mangeat et al. 2003; Mariani et al. 2003; Shindo et al. 2003; Zhang et al. 2003; Suspene et al. 2004; Yu et al. 2004). Its activity is suppressed by HIV-1 Vif, which blocks its virion packaging through protein degradation (Conticello et al. 2003; Kao et al. 2003; Mangeat et al. 2003; Marin et al. 2003; Mehle et al. 2003; Sheehy et al. 2003; Stopak et al. 2003; Yu et al. 2003; Liu et al. 2004). HIV-1 Vif interacts with cellular proteins Cul5, ElonginB, ElonginC, and Rbx1 to form an E3 ubiquitin ligase complex (Yu et al. 2003) similar to ElonginsB/CCul2-SOCS-box (ECS) complexes (Deshaies 1999; Pintard et al. 2004). These cullin-based E3 ligases display striking similarities to Skp1-cullin-F-box (SCF) complexes (Deshaies 1999; Pintard et al. 2004). In both SCF and ECS complexes, Skp1 and ElonginC connect the cullin to the substrate-recognition adaptor protein (F-box and SOCS-box proteins, respectively), binding substrates

[Keywords: Cul5; APOBEC3G; E3 ubiquitin ligase; HIV-1 Vif; ElonginC; SOCS box]

${ }^{4}$ These authors contributed equally to this work.

${ }^{5}$ Corresponding author.

E-MAIL xfyu@jhsph.edu; FAX (410) 614-8263.

Article and publication are at http://www.genesdev.org/cgi/doi/10.1101/ gad. 1250204 through additional distinct protein-protein interaction domains.

It is not clear whether HIV-1 Vif is an integral part of Cul5-ElonginB-ElonginC E3 ubiquitin ligase or merely a linker protein that connects the target protein to the E3 ligase complex. The HIV-1 Vpu protein is known to link the target protein CD4 to a Cull-containing E3 ligase through the F box containing adaptor protein $\beta$ TrCP (Margottin et al. 1998). Marin et al. have observed that the SLQ motif in HIV-1 Vif, which is required for interaction with Cul5-ElonginB-ElonginC (Yu et al. 2003), shares similarity with the SOCS-box motif of SOCS6 (Marin et al. 2003). However, the putative Vif SOCS-box motif lacks a conserved $\mathrm{C}$ that has been shown to be critical for SOCS-box interaction with ElonginC (Aso et al. 1996; Kamura et al. 1998; Lonergan et al. 1998). Therefore, it is not clear whether HIV-1 Vif is a true SOCS-box-containing adaptor protein.

Here, we show that this putative SOCS box in HIV-1 Vif, indeed, mediates its interaction with ElonginC. This novel SOCS-box motif differed from the previously identified SOCS box of cellular proteins, raising the question of the origin of the HIV-1 Vif SOCS box. Unexpectedly, the SOCS-box motif of HIV-1 Vif was necessary but not sufficient for interaction with Cul5-ElonginB-ElonginC. Also, two highly conserved $\mathrm{C}$ residues upstream from the SOCS box in Vif were required to interact with Cul5 but not ElonginC.

\section{Results and Discussion}

The SLQ motif of HIV-1 Vif, which is critical for its assembly with the Cul5-ElonginB-ElonginC complex (Yu et al. 2003), and the downstream amino acids LPXxxxL have significant similarity to the SOCS-box motifs of SOCS6 (Marin et al. 2003) and several other cellular proteins (Kamura et al. 1998; Zhang et al. 1999) that bind ElonginC (Fig. 1A). The structure of the VHLElonginC-ElonginB complex has been determined (Stebbins et al. 1999), and the interface between VHL and ElonginC is well studied. The most critical interaction between the SOCS box of VHL and ElonginC has been mapped to helix 1, loop 10 and helix 3 of VHL (within the SOCS box) and to helix 3, helix 4, loop 5 of ElonginC (Stebbins et al. 1999). L158, C162, V165, and V166 of VHL helix 1, which form a hydrophobic cluster, are important for interaction with ElonginC (Fig. 1B). K159 of VHL is hydrogen-bonded to N108 of ElonginC, which also contributes to the interaction (Fig. 1B). Also, L178 and L184 of VHL form another hydrophobic cluster that stabilizes the interaction between helix 1 of VHL and ElonginC (Fig. 1B).

Amino acids $145-155$ of HIV-1 Vif are predicted to form an $\alpha$-helix. Using the crystal structure of VHLElonginC as a model, L145, A149, A152, and L153 of Vif could form a hydrophobic cluster with spacing similar to L158, C162, V165, and V166 of VHL (Fig. 1C). These hydrophobic residues of HIV-1 Vif are predicted to be on the same face of the $\alpha$-helix and could fit into the hydrophobic pocket of ElonginC. In HIV-1 Vif, Q146 replaces K159 of VHL and has the same potential to form a hydrogen bond with N108 of ElonginC. In fact, Q at this position is more popular in SOCS-box-containing proteins (Kile et al. 2002). L163 and L169 of HIV-1 Vif, like 
A

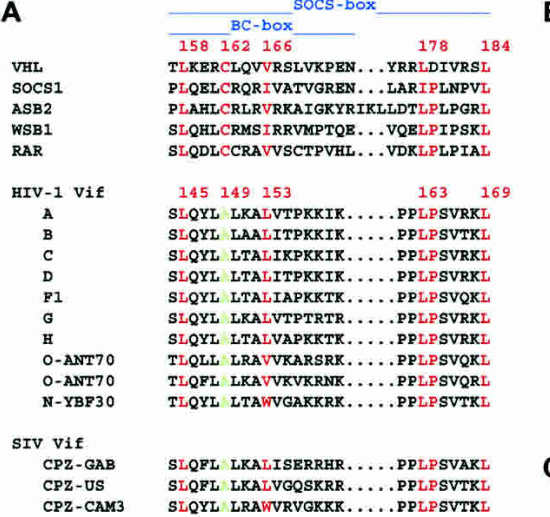

B

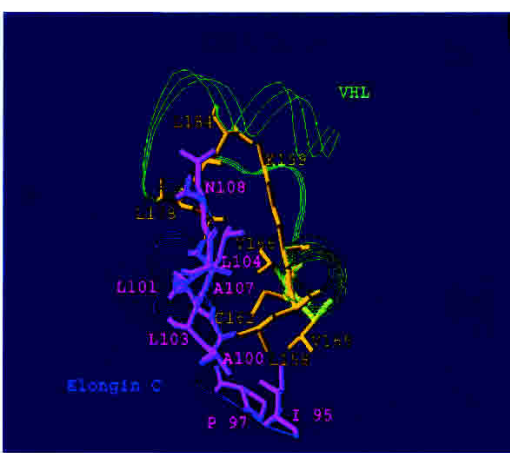

C

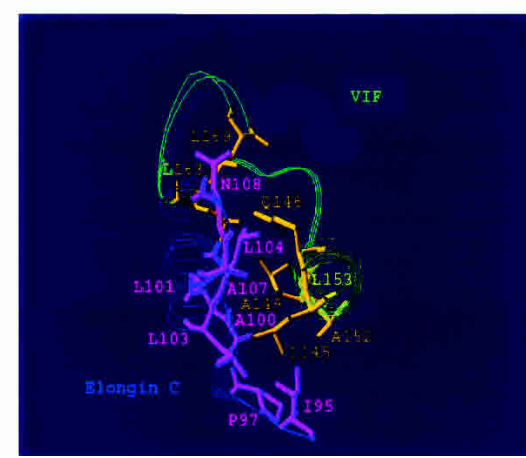

Figure 1. HIV-1 Vif contains a putative SOCS-box motif. (A) Vif of HIV-1 ( $\mathrm{M}, \mathrm{O}$, and $\mathrm{N}$ groups) and closely related SIVcpz viruses contain a SOCS-box-like motif. $(B)$ The interaction interface between VHL and ElonginC was modeled based on structure data from Stebbins et al. (1999). VHL amino acids are in yellow, and those of ElonginC are in cyan. $(C)$ As in the VHL-ElonginC interaction, HIV-1 Vif is predicted to form a hydrophobic interface with ElonginC. Vif amino acids are in yellow, and those of ElonginC are in cyan.

L178 and L184 of VHL, could form another hydrophobic cluster (Fig. 1C) that stabilizes the interaction between Vif and ElonginC. Key features of the SOCS-box-like motifs are highly conserved among all HIV-1 M, N, and O groups as well as the related SIVcpz group from Pan troglodytes troglodytes (Fig. 1A).

A major difference between the SOCS-box-like motifs in HIV-1/SIVcpz Vifs and the consensus SOCS-box motif (Kile et al. 2002) is the lack of a highly conserved C (position C162 in VHL) in Vif, where it is replaced with an A (Fig. 1A). This conserved $\mathrm{C}$ in the consensus SOCS box (C162 in VHL, C554 in ElonginA, and C179 in SOCS1) is critical to interaction with ElonginC (Aso et al. 1996; Kamura et al. 1998; Lonergan et al. 1998). If HIV-1 Vif interacts with ElonginC through this novel SOCS-box motif, one would predict that both $\mathrm{C}$ and $\mathrm{A}$ should be functional and exchangeable at this position. Consistent with this prediction, an A149C substitution did not significantly alter the interaction of VifA149C with ElonginC, ElonginB, and Cul5 (Fig. 2A, lane 4) when compared to wild-type Vif (Fig. 2A, lane 2). Like wild-type Vif, the VifA149C mutant could still interact with h-APO3G (Fig. 2B, lane 3), induce degradation of h-APO3G (Fig. 2C, lane 3), and, more importantly, block the antiviral activity of h-APO3G (Fig. 2D). Both C and A have short side chains, suggesting that this position in the SOCS box prefers amino acids with short side chains. Indeed, when A149 of HIV-1 Vif was changed to another hydrophobic amino acid, L, which has a longer side chain, Vif function was severely impaired. Mutant VifA149L had a reduced ability to form a complex with ElonginC, ElonginB, and Cul5 (Fig. 2A, lane 3). Although

mutant VifA149L remained capable of binding h-APO3G (Fig. 2B, lane 4), it could not induce efficient degradation of h-APO3G (Fig. 2C, lane 4) and had a reduced ability to block h-APO3G function (Fig. 2D). Interestingly, in addition to $\mathrm{A}$ and $\mathrm{C}, \mathrm{S}$ (A149S), which also has a short side chain, could also support Vif function (Fig. 2D). However, any further increase in the size of the side chain (e.g., A149T) significantly decreased HIV-1 Vif function (Fig. 2D).

To further support the argument that Vif can interact with ElonginC through a SOCS-box-like motif, we constructed an ElonginC mutant (EloCs4-HA) in which the critical hydrophobic amino acids of VHL H4 (A100, L101, L103, L104) that interact with the VHL SOCS box (Stebbins et al. 1999| were replaced with hydrophilic S (Fig. 1D). Interaction between the wild-type or mutant ElonginC and HIV-1 Vif in transfected 293 cells was examined by coimmunoprecipitation. As expected, both the wildtype and mutant ElonginC could still bind ElonginB (Fig. 3A, lanes 1,3). Wild-type ElonginC interacted efficiently with HIV-1 Vif (Fig. 3A, lane 1); however, the ElonginC mutant EloC $\Delta 4$-HA had a drastically reduced ability to interact with HIV-1 Vif (Fig. 3A, lane 3). Mutation of only A100 and L103 of ElonginC (EloC $\Delta 2-\mathrm{HA}$ ) also reduced the interaction (Fig. 3A, lane 2). Similarly, mutations of critical residues in the helix region of the Vif SOCS box (SLQ to AAA) that are implicated in binding to ElonginC (Fig. 1E) significantly reduced mutant Vif interaction with ElonginC (Fig. 3B, lane 2). Substitution of the most conserved hydrophobic amino acid, L145, with A alone also reduced the interaction (Fig. 3C, lane 2). This $\mathrm{L}$ residue is conserved in all the SOCS-box motifs (Kile et al. 2002) and is critical for the interaction between ElonginC and the SOCS-box motifs of SOCS1 (Kamura et al. 1998), ElonginA (Aso et al. 1996), and VHL (Lonergan et al. 1998). Although L145A Vif could still interact with h-APO3G (Fig. 3C), it was less able than wild-type Vif to degrade h-APO3G (Fig. 3D, lanes 2,3) and could not effectively block the antiviral activity of h-APO3G (Fig. 3E).

In addition to the BC-box motif (amino acids 145-155), downstream L163 and L169 of the SOCS box were also required for Vif function (Fig. 3E) and efficient interaction with Cul5-ElonginB-ElonginC (Fig. 3F, lanes 2,3). Mutant VifL163S and VifL169S interacted less efficiently with Cul5 than did wild-type Vif (Fig. 3F, lanes $2,3)$ and showed impaired induction of h-APO3G degradation (Fig. 3G). Previous studies have shown that substituting A for L163 and L169 has a lesser effect on HIV-1 Vif function (Simon et al. 1999). L178 and L184, downstream of the VHL SOCS box, form another hydrophobic cluster that stabilizes the interaction between VHL and ElonginC (Stebbins et al. 1999), and L163 and L169 of HIV-1 Vif may play a similar role. L $\rightarrow$ S (hydrophilic) mutations would be more detrimental to this hydropho- 
A

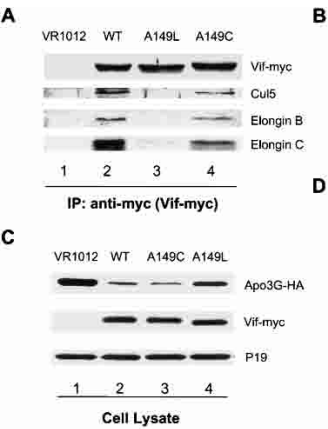

B

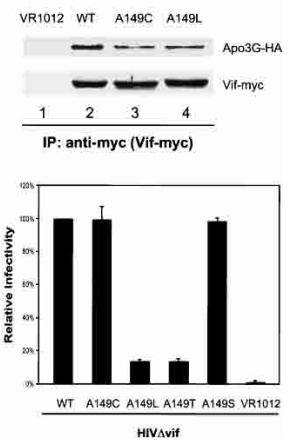

Figure 2. A critical cysteine in the SOCS box is functionally replaced with an alanine in HIV-1 Vif. $(A)$ Cul5-elonginB-elonginC complexes were coimmunoprecipitated with HIV-1 wild-type (WT) Vif (lane 2) and mutant Vif A149C (lane 4) but not mutant Vif A149L (lane 3). (B) Coimmunoprecipitation of h-APO3G with wild-type (lane 2), mutant A149C (lane 3), and mutant A149L Vif (lane 4). (C) Degradation of h-APO3G by wild-type and Vif mutants. 293 cells were cotransfected with phAPO3G-HA plus VR1012, pVif-myc, pVifA149C-myc, or pVifA149L-myc. Equal amounts of cell extract were analyzed by immunoblotting using antibodies against HA for the detection of APOBEC3G, myc for the detection of Vif, and Ribosomal P19 antigen for total protein loading control. $(D)$ Evaluation of Vif function. Viruses were produced from 293/h-APO3G cells cotransfected with HXB2 $\Delta$ Vif plus the control vector VR1012, pHIV-1Vif (wild type [WT]), pVifA149C, pVifA149L, pVifA149T, or pVifA149S. Virus infectivity was examined using MAGI-CCR5 cells. Virus input was normalized by the level of $\mathrm{p} 24$. The infectivity of HXB2 $\Delta$ Vif plus wild-type Vif produced from 293/h-APO3G cells was set as $100 \%$. The results are the average of five independent experiments.

bic interaction than $\mathrm{L} \rightarrow \mathrm{A}$ mutations. Consistent with this idea, we have also observed that L163A has a lesser effect than L163S on HIV-1 Vif function (data not shown).

Direct binding of HIV-1 Vif to ElonginC was also observed for purified recombinant proteins of Vif and ElonginC (Fig. 3H, lane 4), and this interaction was impaired by mutations in the SOCS-box motif (SLQ to AAA) of HIV-1 Vif (Fig. 3H, lane 5).

Interactions between Cul1, Cul2, or Cul3 and Skp1, ElonginC, or MEL-26, respectively, require the N-terminal helix 2 (H2) of these cullins (Zheng et al. 2002; Xu et al. 2004). To examine the involvement of the $\mathrm{N}$-terminal region of Cul5 in ElonginC binding, Cul5 $\Delta \mathrm{N} 1$ and Cul5 $5 \mathrm{~N} 2$ were tested for interaction with ElonginC. Cul5 $\Delta \mathrm{N} 1$ contains a deletion of 32 amino acids (17-48), including H2 of Cul5. Cul5 $\Delta$ N2 contains a 29 (65-93)amino acid deletion downstream from H2. Both Cul5 $\Delta \mathrm{N} 1$ and Cul5 $\Delta \mathrm{N} 2$ were less able than wild-type Cul5 to bind ElonginB and ElonginC (Fig. 4A). In contrast, Rbx1 binding, which requires the C-terminal region of Cul5 (Yu et al. 2003), was not affected by dele-

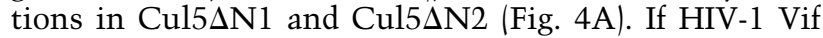
interaction with ElonginC is critical for the formation of the Cul5-ElonginB-ElonginC-Vif complex (Fig. 4B), then Cul5 mutants defective for binding to ElonginC should be less able to interact with Vif. Indeed, both Cul5 $\Delta$ N1 and Cul5 $\Delta \mathrm{N} 2$ were unable to interact efficiently with HIV-1 Vif (Fig. 4C, lanes 5,6). However, the C-terminal regions of Cul5 involved in Nedd8 modification or Rbx1 binding were not required for the interaction between Cul5 and HIV-1 Vif (Fig. 4D). Thus, Cul5 apparently interacts with ElonginC through its $\mathrm{N}$ terminus, and this

interaction is critical for successful Cul5-ElonginBElonginC-Vif complex formation.

Both Cul2 and Cul5 interact with ElonginB and ElonginC to form E3 ubiquitin ligase complexes (Deshaies 1999; Pintard et al. 2004). We have shown that HIV-1 Vif can be coimmunoprecipitated with Cul5 from infected T-cells or transfected 293 cells (Yu et al. 2003). However, it is not clear whether Cul2 can also interact with HIV-1 Vif through ElonginC binding. To answer this question, vectors expressing Cul1, Cul2, Cul3, or Cul5 were cotransfected with HIV-1 Vif into 293 cells. All of the cullins were tagged with an HA epitope and were expressed efficiently in 293 cells (Fig. 5A). Comparable levels of Vif were also detected in the transfected cells (Fig. 5A). All the cullins could be immunoprecipitated by the anti-HA antibody, and HIV-1 Vif was efficiently coimmunoprecipitated with Cul5 (Fig. 5B, lane 4). As expected, Vif was not coimmunoprecipitated with Cul1 (Fig. 5B, lane 1) or Cul3 (Fig. 5B, lane 3), which do not interact with ElonginC (Fig. 5B, lanes 1,3). However,

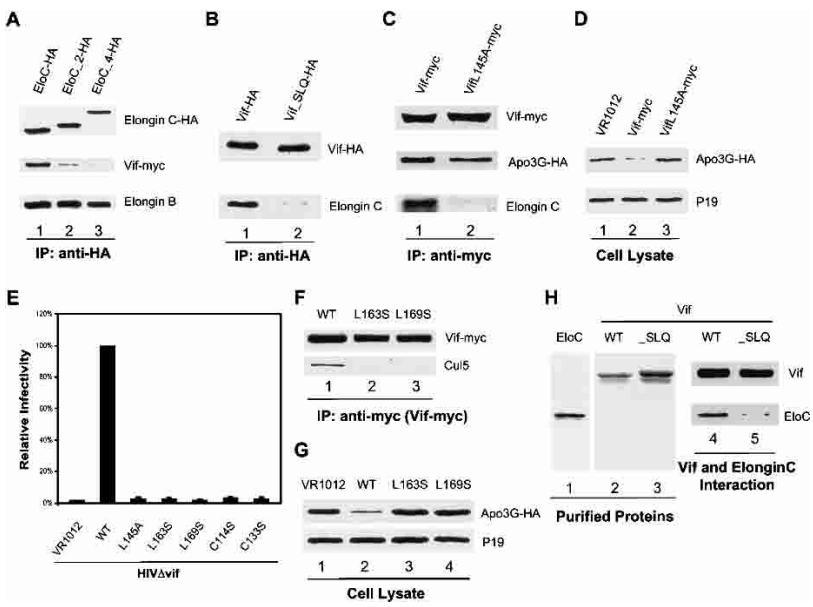

Figure 3. The hydrophobic interface between HIV-1 Vif and ElonginC is critical for their interaction. $(A)$ Interaction of wild-type (WT) and mutant ElonginC with HIV-1 Vif. 293 cells were cotransfected with pHIV-1Vif plus pEloC-HA, pEloC $\Delta 2$-HA, or pEloC $\Delta 4$ HA. Equal amounts of cell extract were immunoprecipitated with anti-HA antibody and analyzed by immunoblotting using antibodies against HA (top panel), Vif (middle panel) or ElonginB (bottom panel). (B) Interaction of ElonginC with wild-type or mutant Vif $\Delta$ SLQ. (C) L145 of HIV-1 Vif is critical for its interaction with ElonginC. 293 cells were cotransfected with phAPO3G-HA plus pVif-myc or pVifL145A-myc. Equal amounts of cell extract were immunoprecipitated with anti-myc antibody and analyzed by immunoblotting using antibodies against Vif (top panel), HA (middle panel), or ElonginC (bottom panel). (D) Reduced degradation of hAPO3G by VifL145A. Equal amounts of cell extract from $C$ were analyzed by immunoblotting using antibodies against $\mathrm{HA}$ for the detection of hAPO3G and Ribosomal P19. (E) Evaluation of Vif function. Virus input was normalized by the level of p24. The infectivity of HXB2 $\Delta$ Vif plus wild-type (WT) Vif produced from 293/h-APO3G cells was set as $100 \%$. The results are the average of three independent experiments. (F) 293 cells were transfected with pVif-myc, pVifL163S-myc, or pVifL169S-myc. Equal amounts of cell extract were immunoprecipitated with anti-myc-tag antibody and analyzed by immunoblotting using antibodies against Vif and Cul5. $(G) \mathrm{Re}-$ duced degradation of hAPO3G by VifL163S or VifL169S. (H) In vitro interaction between ElonginC and Vif or Vif $\Delta$ SLQ. Recombinant GST-ElonginC (lane 1) was incubated with recombinant MBP-Vif (lane 2) or MBP-Vif $\Delta$ SLQ (lane 3) bound to the amylose column. (Top) Bound MBP-Vif (lane 4) or MBP-Vif $\Delta$ SLQ (lane 5) proteins were detected by staining with Coomassie blue. (Bottom) Coprecipitated GST-ElonginC (lanes 4,5) was detected by anti-ElonginC antibody. 
Yu et al.

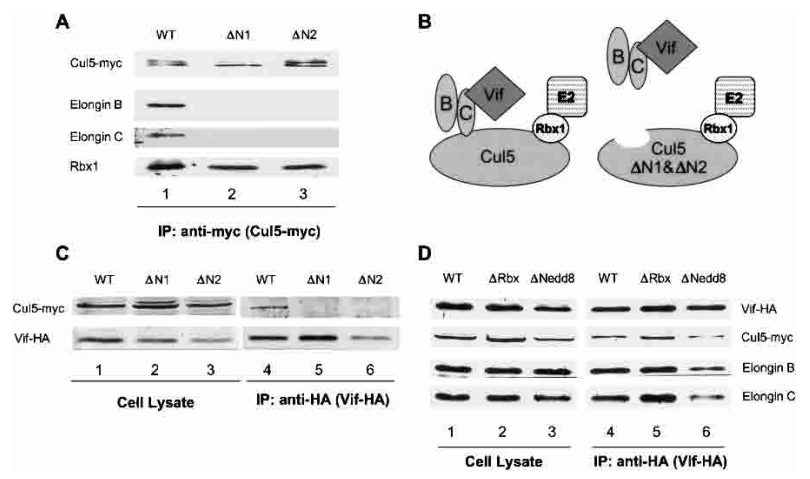

Figure 4. The $\mathrm{N}$-terminal region of Cul5 (including helix 2) is critical for binding to ElonginC and is required to form a complex with HIV-1 Vif. (A) Interaction of ElonginC, ElonginB, and Rbx1 with Cul5, Cul $5 \Delta \mathrm{N} 1$, or Cul5 $\Delta \mathrm{N} 2$. (B) HIV-1 Vif is predicted to form a complex with Cul5 that interacts with ElonginC and ElonginB but not Cul $5 \Delta \mathrm{N} 1$ or Cul5 $\Delta \mathrm{N} 2 .(C)$ Lack of interaction between HIV-1 Vif and Cul5 $\Delta \mathrm{N} 1$ or Cul5 $\Delta \mathrm{N} 2$. (D) Interaction of HIV-1 Vif, ElonginC, and ElonginB with Cul5, Cul5 $\Delta$ Rbx, or Cul $5 \Delta$ Nedd 8 .

coimmunoprecipitation of HIV-1 Vif with Cul2 was also not efficient (Fig. 5B, lane 2) when compared with Cul5 (Fig. 5B, lane 4). Since both ElonginC and ElonginB were coimmunoprecipitated with Cul2 and Cul5 (Fig. 5B, lanes 2,4$)$, these data suggest that HIV-1 Vif selectively recruits Cul5, a finding confirmed by our reverse immunoprecipitation experiments (Fig. 5C): When HIV-1 Vif was coexpressed with Cul1, Cul2, Cul3, or Cul5, only Cul5 was efficiently coimmunoprecipitated with Vif (Fig. 5C, lane 4). As expected, ElonginB and ElonginC were coimmunoprecipitated with Vif in all samples. Therefore, the interaction of HIV-1 Vif with ElonginC alone is not sufficient to explain the selective recruitment of Cul5 but not Cul2.

We suspected that additional regions in Vif may be critical to its interaction with Cul5. Since recruitment of Cul5 is important for Vif function, we focused on the functional essential amino acids of HIV-1 Vif. Two highly conserved C residues in HIV-1/SIVcpz Vifs have been shown to be critical for Vif function (Ma et al. 1994; Simon et al. 1999). Mutation of each residue individually (C114S and C133S) severely impaired the mutant Vif's ability to interact with Cul5 (Fig. 5D, lanes 2,3). Interestingly, VifC114S and VifC133S could still interact with ElonginC and ElonginB, in sharp contrast to the results seen for the SOCS-box mutant VifA149L (Fig. 5D, lane 4). Furthermore, VifC114S and VifC133S remained competent to interact with h-APO3G (Fig. 5E, lanes $2,3,5,6)$ but failed to induce h-APO3G degradation (Fig. $5 \mathrm{~F}$, lanes 3,4 ) and could not suppress h-APO3G activity (Fig. 3E). The $\mathrm{C}$ residues in HIV-1 Vif do not form intramolecular or intermolecular disulfide bonds (Sova et al. 1997). It is less likely that mutations of the two C residues resulted in a general misfolding of HIV-1 Vif, since interactions with both h-APO3G and ElonginC were not affected. Although it is unclear whether these two C residues contribute directly or indirectly to the Vif-Cul5 interaction, these results indicate that regions outside the SOCS box in HIV-1 Vif contribute to Cul5 recruitment into the Cul5-ElonginB-ElonginC-Vif complex.

SOCS boxes have been identified in $>40$ proteins (Kile et al. 2002) that can assemble with Cul2 or Cul5 to form E3 ubiquitin ligases through interaction with ElonginC.
The key binding region in the SOCS box forms a helix (BC box) with a hydrophobic face that interacts with a hydrophobic binding pocket in ElonginC (Stebbins et al. 1999). The consensus BC-box sequence is (S,T,A,P)Lxxx$\operatorname{Cxxx}(\mathrm{L}, \mathrm{I}, \mathrm{A}, \mathrm{V})$. We have shown that although it lacks the highly conserved C residue, HIV-1 Vif uses a SOCS-boxlike motif to interact with ElonginC. This motif could adopt a structure similar to that of the SOCS-box motif in VHL, which binds ElonginC through hydrophobic interfaces. Mutation of critical residues in this motif abolishes the interaction of ElonginC with HIV-1 Vif and therefore the formation of the Cul5-ElonginBElonginC-Vif complex. Mutation of hydrophobic residues in ElonginC that are critical for binding to SOCSbox motifs also abolishes the interaction of ElonginC and HIV-1 Vif. Also, formation of the Cul5-ElonginBElonginC-Vif complex depends on the $\mathrm{N}$-terminal regions of Cul5 that mediate interaction with ElonginC. These results identify the HIV-1 Vif SLQ motif and downstream sequences as a bona fide SOCS-box motif and suggest that HIV-1 Vif is an SOCS-box-containing adaptor protein of the Cul5-ElonginB-ElonginC E3 ubiquitin ligase complex.

A major difference between the BC-box motif of HIV-1 Vif and the consensus BC-box sequence of cellular proteins is the substitution of A for a highly conserved C, which in VHL, ElonginA, and SOCS1 is critical for interaction with ElonginC (Aso et al. 1996; Kamura et al. 1998; Lonergan et al. 1998). Our data indicate that A and C can both be accommodated at this position in HIV-1 Vif and allow it to mediate the interaction with ElonginC and perform its function. Both $\mathrm{A}$ and $\mathrm{C}$ have short side chains. When the A was changed to a bulkier amino acid, L, the mutant Vif A149L lost the ability to efficiently bind ElonginC and induce degradation of h-APO3G, and it was functionally impaired. It appears that the presence of a $\mathrm{C}$ is not as important as the presence of an amino acid with a short side chain at this position. Consistent with this argument is the fact that

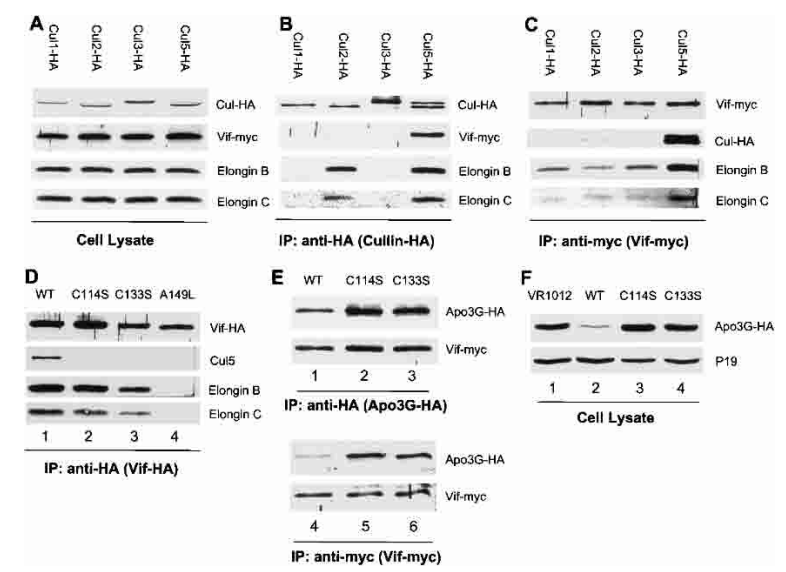

Figure 5. HIV-1 Vif forms a stable complex with Cul5-ElonginBElonginC but not Cul2-ElonginB-ElonginC. (A) Comparable expression of Cul1-HA, Cul2-HA, Cul3-HA, and Cul5-HA as well as Vifmyc, ElonginB, and ElonginC in transfected 293 cells. (B) HIV-1 Vif was coimmunoprecipitated with Cul5-HA but not other cullins. $(C)$ Cul5 but not other cullins was coimmunoprecipitated with HIV-1 Vif. $(D)$ Cul5 was coimmunoprecipitated with wild-type (WT) Vif but not C114S or C133S mutant Vif. (E) Coimmunoprecipitation of h-APO3G with wild-type (WT; lanes 1,4), mutant C114S (lanes 2,5), and mutant C133S Vif (lanes 3,6). (F) Degradation of hAPO3G by wild-type but not C114S or C133S mutant Vif. 
$S$ could also be tolerated at this position but that $T$, with its longer side chain, disrupted Vif function. The reason for this preference for $\mathrm{C}$ in cellular BC-box proteins and $\mathrm{A}$ in HIV-1/SIVcpz Vif is at present unclear. The identification of divergent SOCS-box motifs using A instead of $C$ raises the interesting possibility of the existence of unrecognized cellular or viral proteins containing more divergent SOCS-box motifs. These proteins could also recruit Cul2 or Cul5 through interaction with ElonginC to form E3 ubiquitin ligases and to target proteins for polyubiquitination and degradation.

Both Cul2 and Cul5 use ElonginC to bridge to the SOCS-box adaptor proteins. It is not clear why certain adaptor molecules recruit Cul2, while others, such as HIV-1 Vif and adenovirus E4orf6, prefer Cul5. Our data indicate that additional sequences in adaptor proteins other than the SOCS box may contribute to this selection process. Mutation of the two highly conserved C residues in HIV-1 abolished its interaction with Cul5 without affecting its interactions with ElonginC. The exact sequence motif in HIV-1 Vif involved in the selective recruitment of Cul5 remains to be defined. It is intriguing to consider whether this motif is unique to HIV-1 Vif and whether it could be a novel target for the design of antiviral inhibitors.

\section{Materials and methods}

Plasmid construction

Plasmids HXB2, HXB2 $\Delta$ Vif, VR1012, pHIV-1Vif, pCu15-HA, pCul5-myc, pCul5 $\Delta$ Nedd8, pCul5 $\Delta$ Rbx, and phApo3G-HA have been described (Dettenhofer et al. 2000; Yu et al. 2003). pVifA149C-HA, pVifA149L-HA, pVifA149S-HA, and pVifA149T-HA were made from pVif-HA by changing A149 to C, L, S, and T, respectively. pVifL145A-myc was generated from pVif-myc by changing L145 to A. pVifL163S-myc, pVifL169S, pVifC114S, and pVifC133S were constructed from pVif by changing

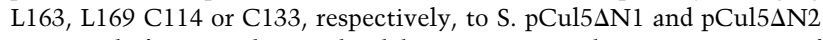
were made from pCul5-myc by deleting amino acids $17-48$ or 65-93 of Cul5. Human ElonginC-HA was amplified by RT-PCR using mRNA from H9 cells: forward primer, 5' -TCTAGAAGGATGGATGGAGAG-3'; and reverse primer, 5'-GGATCCTTACGCATAATCTGGGACGT CATAAGGGTAACAATCTAAGAAGTTCG-3', containing XhoI and BamHI sites, respectively. The PCR product was cloned into VR1012 to generate pEloC-HA. pEloC $\Delta 2$-HA and pEloC $\Delta 4$-HA were made from pEloC-HA by changing either A100 and A103 or A100, L101, L103, and L104 to S. HA-tagged human Cul1, Cul2, and Cul3 were a generous gift of Wade Harper and Jianping Jin (Department of Pathology, Harvard Medical School, Boston, MA).

Cell culture, transfection, MAGI assay, and antibodies

293, 293/h-APO3G, and MAGI-CCR5 cells (Chackerian et al. 1997) were maintained and transfected or infected as previously described (Yu et al. 2003). The antibodies used in this study have been described (Yu et al. 2003): anti-HA antibody-agarose conjugate, anti-myc antibody-agarose conjugate, anti-Vif, anti-Elongin $\mathrm{B}$, anti-Elongin $\mathrm{C}$, anti-Rbx1, anti-myc, anti-HA, and anti-human ribosomal $\mathrm{P}$ antigens.

In vitro interaction

In vitro interaction studies were performed with recombinant GSTElonginC, MBP-Vif, and MBP-Vif $\triangle$ SLQ purified from bacteria. Recombinant fusion proteins were induced and expressed in bacteria, and the crude extracts were applied to a glutathione-Sepharose or amylose column for purification of GST-fusion or MBP-fusion proteins, respectively. The eluted GST or GST-ElonginC were then applied to amylose columnbound MBP-Vif or MBP- Vif $\triangle$ SLQ, respectively. After washing, GSTElonginC proteins were analyzed by immunoblotting.

Immunoprecipitation and immunoblot analysis

Transfected 293T cells were harvested, washed twice with cold PBS, and lysed in lysis buffer (50 mM Tris at $\mathrm{pH} 7.5$, with $150 \mathrm{mM} \mathrm{NaCl}, 1 \%$
Triton X-100, and complete protease inhibitor cocktail tablets) at $4^{\circ} \mathrm{C}$ for $1 \mathrm{~h}$, then centrifuged at $10,000 \times \mathrm{g}$ for $30 \mathrm{~min}$. For myc-tag immunoprecipitation, precleared cell lysates were mixed with anti-myc antibodyconjugated agarose beads (Santa Cruz) and incubated at $4^{\circ} \mathrm{C}$ for $3 \mathrm{~h}$. For HA-tag immunoprecipitation. Precleared cell lysates were mixed with anti-HA antibody-conjugated agarose beads (Roche) and incubated at $4^{\circ} \mathrm{C}$ for $3 \mathrm{~h}$, and then washed three times with washing buffer $(20 \mathrm{mM}$ Tris at $\mathrm{pH} 7.5,100 \mathrm{mM} \mathrm{NaCl}, 0.1 \mathrm{mM}$ EDTA, $0.05 \%$ Tween-20). The beads were eluted with elution buffer $(0.1 \mathrm{M}$ glycine- $\mathrm{HCl}$ at $\mathrm{pH} 3.5$ for anti-myc immunoprecipitation, or $0.1 \mathrm{M}$ glycine- $\mathrm{HCl}$ at $\mathrm{pH} 2.0$ for anti-HA immunoprecipitation). The eluted materials were then analyzed by SDS-PAGE and immunoblotting as previously described (Yu et al. 2003).

\section{Acknowledgments}

We thank R. Garten, T. Sarkis, R. Markham, and Sean Prigge for advice and technical assistance and D. McClellan for editorial assistance. The following reagents were obtained through the AIDS Research Reagents Program, Division of AIDS, NIAID, NIH: antiserum to HIV-1 Vif (Cat. \#2221). This work was supported by a grant from the NIH (AI062644) to X.-F.Y. We declare that we have no competing financial interests.

\section{References}

Aso, T., Haque, D., Barstead, R.J., Conaway, R.C., and Conaway, J.W. 1996. The inducible elongin A elongation activation domain: Structure, function and interaction with the elongin BC complex. EMBO J. 15: $5557-5566$.

Chackerian, B., Long, E.M., Luciw, P.A., and Overbaugh, J. 1997. Human immunodeficiency virus type 1 coreceptors participate in postentry stages in the virus replication cycle and function in simian immunodeficiency virus infection. J. Virol. 71: 3932-3939.

Conticello, S.G., Harris, R.S., and Neuberger, M.S. 2003. The Vif protein of HIV triggers degradation of the human antiretroviral DNA deaminase APOBEC3G. Curr. Biol. 13: 2009-2013.

Deshaies, R.J. 1999. SCF and Cullin/Ring H2-based ubiquitin ligases. Annu. Rev. Cell Dev. Biol. 15: 435-467.

Dettenhofer, M., Cen, S., Carlson, B.A., Kleiman, L., and Yu, X.F. 2000. Association of human immunodeficiency virus type 1 Vif with RNA and its role in reverse transcription. J. Virol. 74: 8938-8945.

Harris, R.S., Bishop, K.N., Sheehy, A.M., Craig, H.M., Petersen-Mahrt, S.K., Watt, I.N., Neuberger, M.S., and Malim, M.H. 2003. DNA deamination mediates innate immunity to retroviral infection. Cell 113: 803-809.

Kamura, T., Sato, S., Haque, D., Liu, L., Kaelin Jr., W.G., Conaway, R.C., and Conaway, J.W. 1998. The Elongin BC complex interacts with the conserved SOCS box motif present in members of the SOCS, ras, WD-40 repeat, and ankyrin repeat families. Genes \& Dev. 12: 3872 3881 .

Kao, S., Khan, M.A., Miyagi, E., Plishka, R., Buckler-White, A., and Strebel, K. 2003. The human immunodeficiency virus Type 1 Vif protein reduces intracellular expression and inhibits packaging of APOBEC3G (CEM15), a cellular inhibitor of virus infectivity. J. Virol. 77: 11398-11407.

Kile, B.T., Schulman, B.A., Alexander, W.S., Nicola, N.A., Martin, H.M., and Hilton, D.J. 2002. The SOCS-box: A tale of destruction and degradation. Trends Biochem. Sci. 27: 235-241.

Lecossier, D., Bouchonnet, F., Clavel, F., and Hance, A.J. 2003. Hypermutation of HIV-1 DNA in the absence of the Vif protein. Science 300: 1112 .

Liu, B., Yu, X., Luo, K., Yu, Y., and Yu, X.F. 2004. Influence of primate lentiviral Vif and proteasome inhibitors on HIV-1 virion packaging of APOBEC3G. J. Virol. 78: 2072-2081.

Lonergan, K.M., Iliopoulos, O., Ohh, M., Kamura, T., Conaway, R.C., Conaway, J.W., and Kaelin Jr., W.G. 1998. Regulation of hypoxiainducible mRNAs by the von Hippel-Lindau tumor suppressor protein requires binding to complexes containing elongins $\mathrm{B} / \mathrm{C}$ and $\mathrm{Cul} 2$. Mol. Cell. Biol. 18: 732-741.

Ma, X.Y., Sova, P., Chao, W., and Volsky, D.J. 1994. Cysteine residues in the Vif protein of human immunodeficiency virus type 1 are essential for viral infectivity. J. Virol. 68: 1714-1720. 
Yu et al.

Mangeat, B., Turelli, P., Caron, G., Friedli, M., Perrin, L., and Trono, D. 2003. Broad antiretroviral defence by human APOBEC3G through lethal editing of nascent reverse transcripts. Nature 424: 99-103.

Margottin, F., Bour, S.P., Durand, H., Selig, L., Benichou, S., Richard, V., Thomas, D., Strebel, K., and Benarous, R. 1998. A novel human WD protein, h- $\beta$ TrCp, that interacts with HIV-1 Vpu connects CD4 to the ER degradation pathway through an F-box motif. Mol. Cell 1: 565-574.

Mariani, R., Chen, D., Schrofelbauer, B., Navarro, F., Konig, R., Bollman, B., Munk, C., Nymark-McMahon, H., and Landau, N.R. 2003. Species-specific exclusion of APOBEC3G from HIV-1 virions by Vif. Cell 114: $21-31$.

Marin, M., Rose, K.M., Kozak, S.L., and Kabat, D. 2003. HIV-1 Vif protein binds the editing enzyme APOBEC3G and induces its degradation. Nat. Med. 9: 1398-1403.

Mehle, A., Strack, B., Ancuta, P., Zhang, C., McPike, M., and Gabuzda, D. 2003. Vif overcomes the innate antiviral activity of APOBEC3G by promoting its degradation in the ubiquitin-proteasome pathway. $J$. Biol. Chem. 279: 7792-7798.

Pintard, L., Willems, A., and Peter, M. 2004. Cullin-based ubiquitin ligases: Cul3-BTB complexes join the family. EMBO J. 23: 1681-1687.

Sheehy, A.M., Gaddis, N.C., Choi, J.D., and Malim, M.H. 2002. Isolation of a human gene that inhibits HIV-1 infection and is suppressed by the viral Vif protein. Nature 418: 646-650.

Sheehy, A.M., Gaddis, N.C., and Malim, M.H. 2003. The antiretroviral enzyme APOBEC3G is degraded by the proteasome in response to HIV-1 Vif. Nat Med. 9: 1404-1407.

Shindo, K., Takaori-Kondo, A., Kobayashi, M., Abudu, A., Fukunaga, K., and Uchiyama, T. 2003. The enzymatic activity of CEM15/Apobec$3 \mathrm{G}$ is essential for the regulation of the infectivity of HIV-1 virion but not a sole determinant of its antiviral activity. J. Biol. Chem. 278: $44412-44416$.

Simon, J.H., Sheehy, A.M., Carpenter, E.A., Fouchier, R.A., and Malim, M.H. 1999. Mutational analysis of the human immunodeficiency virus type 1 Vif protein. J. Virol. 73: 2675-2681.

Sova, P., Chao, W., and Volsky, D.J. 1997. The redox state of cysteines in human immunodeficiency virus type 1 Vif in infected cells and in virions. Biochem. Biophys. Res. Commun. 240: 257-260.

Stebbins, C.E., Kaelin Jr., W.G., and Pavletich, N.P. 1999. Structure of the VHL-ElonginC-ElonginB complex: Implications for VHL tumor suppressor function. Science 284: 455-461.

Stopak, K., de Noronha, C., Yonemoto, W., and Greene, W.C. 2003. HIV-1 Vif blocks the antiviral activity of APOBEC3G by impairing both its translation and intracellular stability. Mol. Cell 12: 591-601.

Suspene, R., Sommer, P., Henry, M., Ferris, S., Guetard, D., Pochet, S., Chester, A., Navaratnam, N., Wain-Hobson, S., and Vartanian, J.P. 2004. APOBEC3G is a single-stranded DNA cytidine deaminase and functions independently of HIV reverse transcriptase. Nucleic Acids Res. 32: 2421-2429.

Xu, H., Svarovskaia, E.S., Barr, R., Zhang, Y., Khan, M.A., Strebel, K., and Pathak, V.K. 2004. A single amino acid substitution in human APOBEC3G antiretroviral enzyme confers resistance to HIV-1 virion infectivity factor-induced depletion. Proc. Natl. Acad. Sci. 101: 56525657.

Yu, X., Yu, Y., Liu, B., Luo, K., Kong, W., Mao, P., and Yu, X.F. 2003. Induction of APOBEC3G ubiquitination and degradation by an HIV-1 Vif-Cul5-SCF complex. Science 302: 1056-1060.

Yu, Q., Konig, R., Pillai, S., Chiles, K., Kearney, M., Palmer, S., Richman, D., Coffin, J.M., and Landau, N.R. 2004. Single-strand specificity of APOBEC $3 \mathrm{G}$ accounts for minus-strand deamination of the HIV genome. Nat. Struct. Mol. Biol. 11: 435-442.

Zhang, J.G., Farley, A., Nicholson, S.E., Willson, T.A., Zugaro, L.M., Simpson, R.J., Moritz, R.L., Cary, D., Richardson, R., Hausmann, G., et al. 1999. The conserved SOCS box motif in suppressors of cytokine signaling binds to elongins $\mathrm{B}$ and $\mathrm{C}$ and may couple bound proteins to proteasomal degradation. Proc. Natl. Acad. Sci. 96: 2071-2076.

Zhang, H., Yang, B., Pomerantz, R.J., Zhang, C., Arunachalam, S.C., and Gao, L. 2003. The cytidine deaminase CEM15 induces hypermutation in newly synthesized HIV-1 DNA. Nature 424: 94-98.

Zheng, N., Schulman, B.A., Song, L., Miller, J.J., Jeffrey, P.D., Wang, P., Chu, C., Koepp, D.M., Elledge, S.J., Pagano, M., et al. 2002. Structure of the Cul1-Rbx1-Skp1-F boxSkp2 SCF ubiquitin ligase complex. $\mathrm{Na}$ ture 416: 703-709. 


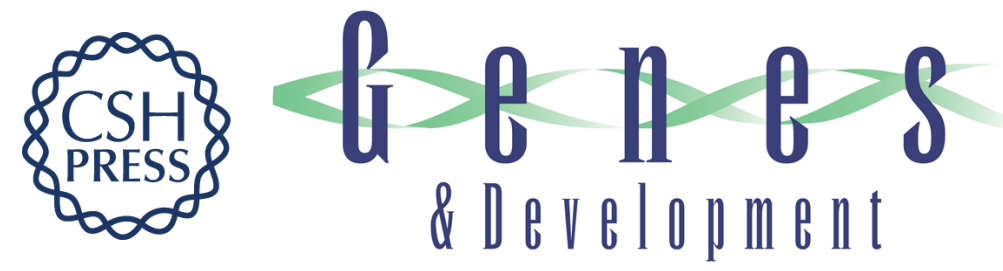

\section{Selective assembly of HIV-1 Vif-Cul5-ElonginB-ElonginC E3 ubiquitin ligase complex through a novel SOCS box and upstream cysteines}

Yunkai Yu, Zuoxiang Xiao, Elana S. Ehrlich, et al.

Genes Dev. 2004, 18:

Access the most recent version at doi:10.1101/gad.1250204

\section{License}

Email Alerting

Receive free email alerts when new articles cite this article - sign up in the box at the top

Service right corner of the article or click here.

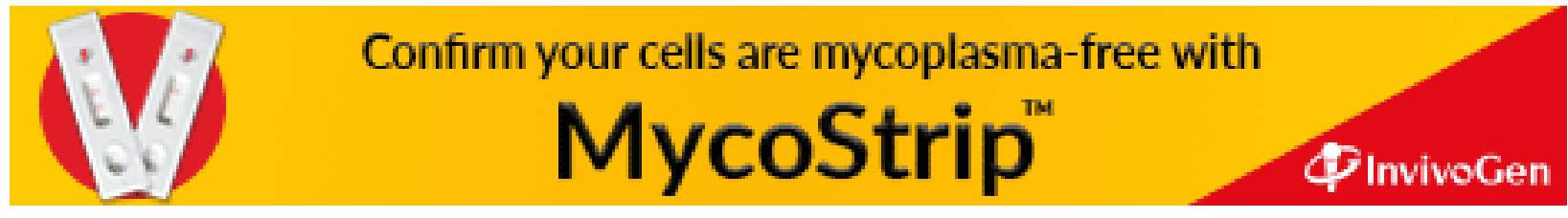

